\title{
Pub: Probing the Interaction of Human Salivary Alpha-Amylase and Amylase Binding Protein A (AbpA) of Streptococcus gordonii
}

\author{
Prerna Gopal, Chandran Ragunath, Vishal Vyas, Mayilvahanan Shanmugam and Narayanan Ramasubbu*
}

Department of Oral Biology, Rutgers School of Dental Medicine, 185 South Orange Ave, Newark NJ 07103, USA

\begin{abstract}
Amylase binding protein $\mathrm{A}(\mathrm{AbpA})$ of Streptococcus gordonii serves as a major receptor for human salivary a-amylase (HSAmy), the predominant enzyme in human salivary secretions, to bind to the bacterial cell surface. On enamel surfaces, the binding of AbpA to HSAmy renders S. gordonii act as a scaffold for other oral bacteria to attach to the acquired enamel pellicle leading to complex bacterial communities and invasion of host tissues. While the role of $\mathrm{AbpA}$ in adhesion, starch metabolism and biofilm formation in influencing the ecology of the oral biofilms has been established, the structure function relationships of AbpA are yet to be defined. Since distally located aromatic residues of HSAmy are involved in the interaction with $A b p A$, we hypothesized that AbpA might use separate structural regions to bind to HSAmy. To test this, several deletion mutants of AbpA were constructed and studied to correlate the effect of deletions in binding to HSAmy in the following: 1) their secondary structural features through circular dichroism studies; 2) their ability to alter the capacity of HSAmy to hydrolyze starch and several oligosaccharides after complex formation and 3) their binding to HSAmy using surface plasmon resonance spectroscopy. Our results showed that a) $\mathrm{AbpA}$ does not bind at the active site of salivary $\alpha$-amylase; b) both the N-terminal region (24-56 residues) and a central region (residues 124-165) are required for binding to HSAmy; and c) the C-terminal end residues (166- 195) are not necessary for binding. These results clearly show that AbpA binding to HSAmy encompasses distinct and distal regions of the structure.
\end{abstract}

Keywords: $a$-amylase; Mutagenesis; Hydrolysis; Bacterial binding; Amylase-binding

Abbreviations: HSAmy: Human Salivary a-amylase; AbpA: Amylase Binding Protein A; SPR: Surface Plasmon Resonance; RU: Resonance Unit

\section{Introduction}

Human salivary a-amylase (HSAmy), a multifunctional enzyme present in the oral cavity of humans, plays a significant role in salivabacteria interactions and participates in the establishment of dental biofilm [1]. HSAmy is consistently present in the enamel pellicle [2] where it acts as a receptor to a group of streptococci known as the amylase-binding streptococci (ABS), which include S. gordonii, $S$. mitis, S. parasanguis, S. cristatus and S. salivarius [3-6]. Among these bacteria, $S$. gordonii, is a pioneer colonizer present in substantial quantity in the early enamel pellicle partly due to its ability to attach to HSAmy present in the pellicle. This initial attachment to HSAmy might be a critical step in the development of dental biofilms since it provides a scaffold for non-amylase binding streptococci. Nutritional support for the dental biofilm bacteria is provided by HSAmy through its ability to hydrolyze $\alpha$-1,4-glucosidic bonds in starch and other polysaccharides during food intake. Thus, each of the three functions of the enzyme, namely, hydrolysis of starch, adherence to enamel and binding to viridans streptococci together might play a role affecting the formation of oral biofilms.

The ability of S. gordonii to bind to HSAmy has been attributed to the presence of low- molecular weight protein $(\mathrm{AbpA})$ and high molecular weight protein (AbpB) [7-9]. Recent studies have shown that several streptococci express a number of proteins of different sizes ranging from 20 to $87 \mathrm{kDa}$ with a common property of binding to HSAmy. Amongst these amylase-binding proteins, the low molecular weight protein AbpA has been extensively studied to establish its role in $S$. gordonii biofilm formation [10-12]. Several characteristics of AbpA have led to a detailed study of this protein including its small size, its role in biofilm formation of $S$. gordonii, its ability to bind HSAmy to the bacterial surface and thus enhance starch-induced growth [11]. Its ability to increase the enzyme activities with Gtfs of both S. gordonii and
S. mutans in the presence of HSAmy have led to suggest that HSAmy: AbpA complex may modulate the bacterial adhesion and colonization in ways that is not completely understood $[1,12]$. The second protein $\mathrm{AbpB}$, due to its enzymatic property of being a dipeptidase, although important for bacterial colonization, is somewhat limited in the binding of HSAmy to the bacterial surface [1]. Interestingly, mutation of $a b p B$ did not abolish the binding of HSAmy whereas mutation of $a b p A$ led to the absence of HSAmy binding to S. gordonii [13]. Unlike AbpA, the role of $\mathrm{AbpB}$ has been in bacterial colonization suggested to be through nutrient acquisition pathways [1].

While significant progress has been made in the identification of various amylase-binding proteins of oral streptococci and study of their functions, especially AbpA, little is known about the structural basis for these proteins to interact with HSAmy. In this regard, several studies have been reported on the structural aspects of HSAmy and the role of several residues in the active site as well as in the secondary saccharide binding sites [14-20]. Interestingly, our earlier study suggested that the AbpA interacts with amylase over an extended surface region with two distinct sites both of which have aromatic residues [20]. The binding occurs in such a way that the binding of smaller oligosaccharides to the active site in HSAmy is not blocked but hinders the binding of starch suggesting that secondary saccharide binding sites of HSAmy (site involving W203 and W284) in the vicinity of the active site were being used by AbpA [20]. Nevertheless, for a complete understanding of the binding interaction between HSAmy and AbpA, structure- function

*Corresponding author: Narayanan Ramasubbu, Department of Oral Biology C-634, MSB, UMDNJ, 185 South Orange Ave, Newark, NJ 07103, USA, Tel: (973) 972-0704; Fax: (973) 972-0045; E-mail: ramasun1@sdm. rutgers. edu

Received July 10, 2013; Accepted August 20, 2013; Published August 23, 2013

Citation: Gopal P, Ragunath C, Vyas V, Shanmugam M, Ramasubbu N (2013) Pub: Probing the Interaction of Human Salivary Alpha-Amylase and Amylase Binding Protein A (AbpA) of Streptococcus gordonii. Mol Biol 2: 111. doi:10.4172/21689547.1000111

Copyright: $\odot 2013$ Gopal P, et al. This is an open-access article distributed under the terms of the Creative Commons Attribution License, which permits unrestricted use, distribution, and reproduction in any medium, provided the original author and source are credited. 
Citation: Gopal P, Ragunath C, Vyas V, Shanmugam M, Ramasubbu N (2013) Pub: Probing the Interaction of Human Salivary Alpha-Amylase and Amylase Binding Protein A (ABPA) of Streptococcus Gordonii. Mol Biol 2: 111. doi:10.4172/2168-9547.1000111

Page 2 of 6

studies on AbpA are warranted. In this regard, it was necessary to understand the structural features of AbpA that could provide such an extended interaction with $\alpha$-amylase. Hence, in this study, we have focused on the structure function relationships of AbpA and its interaction with HSAmy through molecular modeling, selective deletion mutagenesis and biophysical studies.

\section{Materials and Methods}

\section{General procedures}

All the chemicals used herein were obtained from Sigma Chemical Co. Oligonucleotides used in this study were obtained from Integrated DNA Technologies. The core facilities at the University of Medicine and Dentistry of New Jersey were used for nucleotide sequencing.

\section{Bacterial strains, plasmids and growth conditions}

S. gordonii G9B was used for cloning of abpA gene, E. coli DH5a $($ NEB10- $\beta)$, E. coli Rosetta ${ }^{\mathrm{TM}}$ (DE3) and vector pET29b were used for standard cloning and protein expression. Streptococci were maintained on tryptic soy broth supplemented with $0.5 \%$ yeast extract (TSBY) and were incubated at $37^{\circ} \mathrm{C}$ for 12 to 16 hours. E. coli strains were grown under aerobic conditions with shaking for 12 to $16 \mathrm{~h}$ at $37^{\circ} \mathrm{C}$ in Luria-Bertani (LB) broth and maintained on LB agar. E. coli strains containing recombinant clones were plated on LB agar and supplemented as required with kanamycin $(30 \mu \mathrm{g} / \mathrm{ml})$. The $a b p A$ gene was PCR amplified from the $S$. gordonii G9B genomic DNA using a forward primer that introduced an NdeI restriction site and a reverse primer containing a KpnI restriction site and a stop codon. The PCR product, double digested with enzymes NdeI and KpnI, was ligated into the pET29b vector (Novagen) between its NdeI and KpnI restriction sites to yield the plasmid pCR4. The resulting plasmid encodes the fulllength mature protein $\left(\mathrm{AbpA}^{24-195}\right)$ with a C-terminal His ${ }_{6}$ tag. Selective deletion of $\mathrm{N}$ and $\mathrm{C}$ terminal domain was done to generate four variants of AbpA. The full length AbpA without the signal sequence was named PG1 (24-195), N terminal deletion variant was named PG2 (54-195), C terminal deletion was named PG3 (24- 165), a truncated mutant with the 1-100 residues of the mature protein was named PG4 (24-124) and a fifth mutant with residues encompassing the C-terminal region (residues 124-195) was named PG5. The primers used for construction of the full length AbpA and the variants are listed in Table 2.

\section{Expression and purification of AbpA}

The protein AbpA was expressed using E. coli Rosetta ${ }^{\mathrm{Tw}}$ (DE3) cells transformed with pCR4 using procedures described before [20]. Typically, $1 \mathrm{~L}$ of Luria-Bertani broth supplemented with $30 \mu \mathrm{g} / \mathrm{mL}$ of kanamycin per $\mathrm{mL}$ was inoculated with $10 \mathrm{~mL}$ of an overnight culture of E. coli strain Rosetta ${ }^{\text {ma }}$ (DE3) (Novagen) [21] transformed with the plasmid pCR4. After incubation at $37^{\circ} \mathrm{C}$ for $4 \mathrm{~h}$ or until the optical density of the culture (OD 600) reached 0.6 , protein expression was induced by the addition of isopropyl- $\beta$ D-1-thiogalactopyranoside to a final concentration of $0.1 \mathrm{mM}$. The cells were incubated post-induction for $5 \mathrm{~h}$ at $37^{\circ} \mathrm{C}$ and then harvested by centrifugation at $\times 6000 \mathrm{~g}$. The cell pellet was resuspended in $20 \mathrm{~mL}$ of lysis buffer $(20 \mathrm{mM}$ Tris- $\mathrm{HCl}$, pH 8. 0, $500 \mathrm{mM} \mathrm{NaCl}, 1 \mathrm{mM}$ phenylmethylsulfonyl fluoride and $0.1 \%$ Nonidet P-40). The cell suspension was then sonicated on ice for $30 \mathrm{~s}$ (x5 with 2 min intervals) at 30\% capacity with a 30\% duty cycle by using a Branson model 450 sonicator equipped with a microprobe. The cell debris was pelleted by centrifugation $\times\left(15,000 \mathrm{~g}\right.$ for $\left.20 \mathrm{~min} ; 4^{\circ} \mathrm{C}\right)$, and the supernatant was filtered using $0.45 \mu \mathrm{m}$ syringe filter (Corning) and purified by AKTA $^{*}$ FPLC system controlled by UNICORN ${ }^{\mathrm{rw}}$ software

\begin{tabular}{|c|c|c|c|}
\hline Analyte & $\mathbf{k}_{\mathbf{a}}(\mathbf{1} / \mathbf{M s})$ & $\mathbf{k}_{\mathbf{d}}(\mathbf{1 / s})$ & $\mathbf{K}_{\mathbf{A}}(\mathbf{1} / \mathbf{M})$ \\
\hline $\mathrm{AbpA}^{24-195}$ (PG1) & $5.7 \times 10^{4}$ & $8.0 \times 10^{-6}$ & $7.1 \times 10^{9}$ \\
\hline $\mathrm{AbpA}^{54-195}$ (PG2) & $1.6 \times 10^{4}$ & $2.0 \times 10^{-2}$ & $8.2 \times 10^{5}$ \\
\hline $\mathrm{AbpA}^{24-195}$ (PG3) & $2.1 \times 10^{5}$ & $1.4 \times 10^{-4}$ & $1.2 \times 10^{-6}$ \\
\hline $\mathrm{AbpA}^{24-195}$ (PG4) & $\mathrm{ND}$ & $\mathrm{ND}$ & $1.4 \times 10^{9}$ \\
\hline
\end{tabular}

Table 1: Kinetic parameters for the binding of AbpA to HSAmy enzymes derived from surface plasmon resonance studies.

\begin{tabular}{|c|c|c|}
\hline No. & Variant & Primers \\
\hline PG1 & Full length without the signal sequence & $\begin{array}{c}\text { AbpA/Ndel-24/F } \\
\text { 5'GGAATTCCATATGGCTGATGAAGCTACTGATGCAGC3' } \\
\text { AbpA/Kpnl-195/R } \\
\text { 5'CGGGGTACCTTAGTGGTGGTGGTGGTGTGTTTAACTGCTGAAGTT } \\
\text { TTAACTGCTGAAGTTTTTGGAAGTGC 3' }\end{array}$ \\
\hline PG2 & $30 \mathrm{~N}$ terminal deletion $(54-195)$ & $\begin{array}{c}\text { AbpA/Ndel-54/F } \\
\text { 5'GGAATTCCATATGTTGGCTCAACATGATGGTGAAATCCG3' } \\
\text { AbpA/Kpnl-195/R } \\
\text { 5'CGGGGTACCTTAGTGGTGGTGGTGGTGGTGAGCAGGAGTAGTAG } \\
\text { CTCCTTTGTC3' }\end{array}$ \\
\hline PG3 & $30 \mathrm{C}$ terminal deletion $(24-165)$ & $\begin{array}{c}\text { AbpA/Ndel-24/F } \\
\text { 5'GGAATTCCATATGGCTGATGAAGCTACTGATGCAGC3' } \\
\text { AbpA/Kpnl-165/R } \\
\text { 5'CGGGGTACCTTAGTGGTGGTGGTGGTGGTGAGCAGGAGTAGCTC } \\
\text { CTTTGTC-3' }\end{array}$ \\
\hline PG4 & 1st 100 amino acids $(24-124)$ & $\begin{array}{c}\text { AbpA/Ndel-24/F } \\
\text { 5'GGAATTCCATATGGCTGATGAAGCTACTGATGCAGC3' } \\
\text { AbpA/Kpnl-124/R } \\
\text { 5'CGGGGTACCTTAGTGGTGGTGGTGGTGGTGTTCTGTAGCGTTGTT } \\
\text { GTATGTAGC3' }\end{array}$ \\
\hline PG5 & Last 70 amino acids $125-195$ & $\begin{array}{c}\text { AbpA/Ndel-125/F } \\
\text { 5'GGAATTCATATGCAAGAAGGCAAAACATACATCCAAGG3' } \\
\text { AbpA/Kpnl-195/R } \\
\text { 5'CGGGGTACCTTAGTGGTGGTGGTGGTGGTGTTTAACTGCTGAAGT } \\
\text { TTTTGGAAGTGC3' }\end{array}$ \\
\hline
\end{tabular}

Table 2. Primers used in this study to generate AbpA variants. The underlined sequences correspond to the restriction sites (Ndel in forward and Kpnl in reverse). All constructs have a $\mathrm{His}_{6}$.tag sequence at the 3 ' end. 
version 5. 1 (GE Healthcare) as previously described for other proteins purified from our laboratory. The presence of the AbpA protein was determined by SDS-PAGE and Coomassie Blue staining. The purity and molecular mass of AbpA protein was determined by mass spectral analysis. The four variants of AbpA were expressed and purified essentially using the procedures established for the mature protein. The truncated variants also contained a His6 tag at their respective C-terminal end.

\section{Bioinformatics}

A model of AbpA was derived from in silico analysis. The amino acid sequence of AbpA was submitted to the HHpred server [22] for detecting proteins with remote homology and predicting a structure. This method is based on generating an alignment of homologs by multiple iterative searches using PSI-BLAST against NCBI nonredundant database. This database search is combined with predicted secondary structure and confidence values from PSIPRED [23]. Following the iterative procedure, a profile hidden Markov model (HMM) for AbpA was constructed that included the information about predicted secondary structure for the input sequence. The query HMM thus generated was then compared with HMMs from the Protein Data Bank (PDB) by the HHsearch software [22]. HHsearch uses an algorithm that uses position- specific gap penalties and scores for similarity in the secondary structure to construct local alignments with the query sequence $(\mathrm{AbpA})$ and the matching database sequences. The best- matched proteins from the PDB database were selected as templates for comparative modeling with the MODELLER software [24]. The resulting structure models were viewed with PyMOL version 1. $0[25]$.

\section{Circular dichroism}

CD spectra were recorded on an AVIV model 400 spectrophotometer equipped with a five-position thermostated cell holder controlled by a Hewlett-Packard Peltier temperature controller $\mu \mathrm{g} / \mathrm{mLin}$. HSAmy and AbpA solution of $100 \mu \mathrm{g} / \mathrm{ml}$ in $0.15 \mathrm{M} \mathrm{NaCl}$ and $0.01 \mathrm{M}$ sodium phosphate $(\mathrm{pH}$ 7.4) was transferred into quartz cells with $0.1 \mathrm{~cm}$ path length. Concentrations were determined using a protein assay kit (Bio-Rad) and nanodrop. Spectra were collected from $260 \mathrm{~nm}$ to approximately $200 \mathrm{~nm}$ (with a step size of $0.5 \mathrm{~nm}$ and a bandwidth of $1.5 \mathrm{~nm}$ ) at room temperature, corrected for buffer baseline, and measured in the same cells. These results were normalized to mean residue ellipticities $([\theta])$.

\section{Enzyme activity and other in vitro assays}

The AbpA and its variants with their truncations might be expected to exhibit differences in their ability to bind to HSAmy. Previous studies [20] have shown that residual HSAmy activity could be determined from the supernatants of mixtures in which HSAmy was incubated with AbpA on ice for 30 minutes. Typical experiments were carried out using $1 \%$ soluble starch as substrate at $25^{\circ} \mathrm{C}$ for $5 \mathrm{~min}$ in $20 \mathrm{mM}$ phosphate buffer ( $\mathrm{pH}$ 6.9) containing $6 \mathrm{mM} \mathrm{NaCl}$. Prior to use, the HSAmy solution $(150 \mathrm{nM})$ was incubated with various concentrations of the AbpA variants $(0-1.6 \mu \mathrm{M})$ for 30 minutes on ice to allow for complex formation after which an aliquot of the reaction mixture was used in the dinitrosalicylic acid assay [Bernfeld, 1955]. Similarly, to test for the residual activity in the presence of smaller oligosaccharides, enzymatic reactions were carried out using maltoheptaose (G7; Boehringer Mannheim). Each reaction mixture (total volume of $100 \mu \mathrm{l})$ consisted of HSAmy $(60 \mathrm{nM})$, AbpA variants $(480 \mathrm{nM})$, maltoheptaose $(0.5 \mathrm{mM})$. As with the starch assay, HSAmy and AbpA variants were incubated for 30 minutes before G7-PNP was added. This reaction mixture was injected onto the HPLC column 5 min after the oligosaccharide was added and the product distribution of amylase and amylase bound to AbpA variants were determined by HPLC analyses using a chromatography system (Varian) as described before [20]. The amount of residual G7 was determined using the area under each peak and converting these into concentrations using the standard curves. The product profile was analyzed based on retention times of standards run under similar conditions without the addition of the enzyme. The concentrations of the HSAmy and the AbpA variants were determined by a protein assay kit (Bio-Rad) using bovine serum albumin (BSA; Sigma Chemical Co. ) as the standard. All experiments were performed in triplicate and repeated twice.

\section{BIAcore assays}

Surface plasmon resonance experiments were performed on a BIAcore X 2000 instrument equipped with research-grade CM5 sensor chips as detailed before [20] with some minor modifications. The amylase binding protein constructs PG1 through PG4 were immobilized on the chip using the amine coupling kit from BIAcore. Briefly, the sensor chips were activated with a solution containing $0.1 \mathrm{M}$ N-hydroxysuccinimide and 0. 4 M 1-ethyl-3-(3-dimethylaminopropyl) carbodiimide (1:1). Typically, AbpA were immobilized to a maximum value $1000 \mathrm{RU}$ wherein the change in refractive index induced by the association of compounds interacting with the sensor surface is expressed in RU. Unreacted residual N-hydroxysuccinimide esters were inactivated using $1 \mathrm{M}$ ethanolamine. Real-time binding analyses to $\mathrm{AbpA}$ variants were performed at $25^{\circ} \mathrm{C}$ using HSAmy in a buffer containing $10 \mathrm{mM}$ HEPES (pH 6. 9), $150 \mathrm{mM} \mathrm{NaCl}$ and $2.5 \mathrm{mM} \mathrm{CaCl}$ at varying concentrations ranging from 0.125 to $1 \mu \mathrm{M}$ at a flow rate of $20 \mu \mathrm{L} / \mathrm{min}$ at $25^{\circ} \mathrm{C}$. After each experiment, the chip surface was regenerated using $10 \mathrm{mM} \mathrm{HCl}$. The binding parameters (kon, koff, $\mathrm{Ka}$, and $\mathrm{Kd}$ ) for the interaction were calculated using BIAevaluation 3. 2 software. The curves were fitted according to a simple 1:1 interaction. The fitting residuals did not exhibit any significant systematic deviations and were minimal (-1 to 1$)$.

\section{Results}

\section{Model of AbpA and design of the AbpA mutants}

The mature AbpA is 172 residues long (24- 195 numbering) excluding the signal sequence. Since the crystal structure of AbpA is not yet available and has been difficult to achieve, we resorted to the molecular modeling analysis. The homology model of AbpA obtained through the HHpred server revealed a structure with a large content of $\alpha$-helical segments (Figure 1). It should be emphasized that the model presented here may not be perfectly accurate since it is based on bioinformatics approach. However, our approach has some merits to further the structural knowledge of AbpA in the absence of a definitive structure of $\mathrm{AbpA}$ through either $\mathrm{x}$-ray crystallographic techniques or NMR. The significant feature in this model is the preponderance of the helical structure adopted by the molecule especially at the $\mathrm{N}$-terminal end of the molecule. The C-terminal end of the molecule (residues $165-195)$ is comprised of both helix as well as $\beta$-strands. The model obtained, with a caveat that it could be somewhat less reliable due to paucity of significant homologous structures, could be easily tested for the preponderance of helical regions using circular dichroism, which is described below. Using this tentative model, the truncated mutants were designed with the following rationale. The mutant PG2, which lacks the first 30 residues (residues 24-53) were designed to test the role of the unordered $\mathrm{N}$-terminal segment and the first small helical segment shown in red (Figure 1). The mutant PG3 is lacking the last 30 residues (residues 165-195), which also has a helical segment (blue 


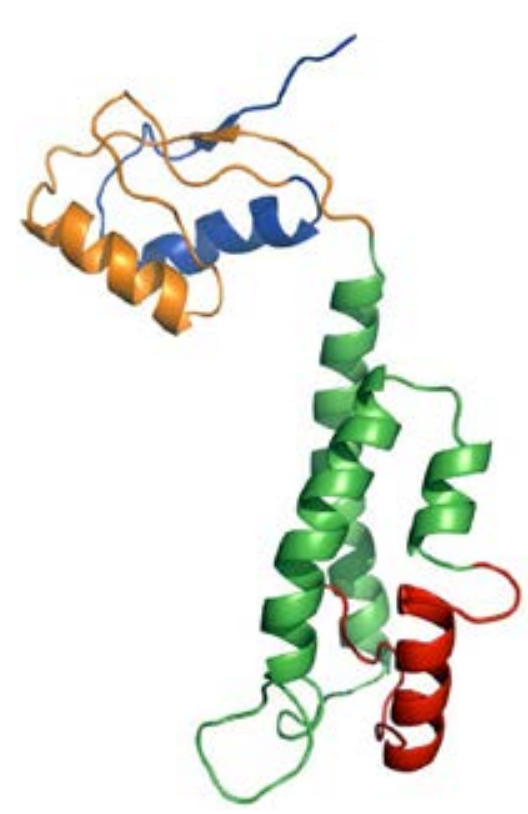

Figure 1: Homology modeling of AbpA. HHpred prediction of AbpA protein rendered using PyMOL [25]. The color coded segments refer to the various deletion segments designed in this study. Red, N-terminal 30-residues (PG2); Blue, C-terminal 30 residues (PG3); and Orange, additional 30 residues in the C-terminal domain (PG4).

segment in Figure 1). The mutant, PG4, was designed such that it lacked the C-terminal domain harboring two helices and the $\beta$-strands (blue and orange segments). Finally, the mutant PG5, was constructed such that it contained only the $\mathrm{C}$-terminal domain.

\section{Characterization and purification of $\mathrm{AbpA}$ variants}

The peptide sequence of the full length AbpA from $S$. gordonii G9B was identical to the sequence of AbpA from $S$. gordonii Challis obtained from the Oralgen database (http://www. stdgen. lanl. gov/) except for at position 89 which is an Arg in G9B vs. Gln in Challis. SDS PAGE gels of the resulting purified protein stained with Coomassie Blue showed a single band of approximately $20 \mathrm{kDa}$ consistent with the calculated mass of the protein. Similarly, the other truncated variants also showed corresponding reduction in sizes in the SDS-PAGE gels (data not shown). AbpA mutant proteins were obtained at greater than $99 \%$ purity, with a cell culture yield of $10-15 \mathrm{mg} / \mathrm{L}$.

\section{Secondary structure content of AbpA variants}

It is conceivable that the overall conformation of truncated variants could have altered due to the large deletions in the structure. We therefore, measured the structural characteristics of the AbpA proteins using circular dichroism (CD) spectroscopy to determine whether or not gross conformational changes have occurred in any of the mutants. As can be seen in Figure 2, the mutants PG2, PG3 and PG4 retained the characteristics of mature AbpA (PG1) with ordered secondary structures reminiscent of the helical structures. Notably, all these variants except PG5, has the characteristic double minima of a typical $\alpha-h$ helical protein. The CD spectrum of PG5, however, resembles a typical unordered structure, likely due to the large deletion in the sequence (residues 24-123), which represents a large fraction of the helical structures. These CD spectra partly validate the model obtained by the homology modeling since both PG1 and PG2 have very similar spectra whereas PG3 and PG4 have a larger negative minimum at 222 $\mathrm{nm}$. The increase in helicity in these two variants could have arisen from the removal of either unordered regions (PG3) or a higher ratio of helix vs. other structures in PG4. The estimation of the secondary structure content from the spectra of these variants was analyzed using the program suite CDPRO. The values estimated by the individual programs were comparable and suggest that these variants are mostly helical structures.

\section{Starch hydrolysis studies}

Previous studies have shown that AbpA forms a tight complex with HSAmy and the formation of the complex reduces the HSAmy's ability to hydrolyze starch [20]. To determine whether or not the binding of AbpA variants also results in the reduction of starch hydrolyzing activity of HSAmy, the four variants along with the full length AbpA were tested. After binding, the starch hydrolysis by unbound Amylase was studied. The results are shown in figure 3. As has been observed previously, the full length AbpA, PG1 showed the highest binding as the starch hydrolysis is reduced by increasing concentration of AbpA. Interestingly, partial deletion of the C-terminal domain, as in PG3, did not have any effect in the starch hydrolysis. The residual activity of PG3: HSAmy complex mirrored that of the PG1: HSAmy complex suggesting that the last 30 residues may or may not bind to HSAmy in a way that restricts starch binding. In cases of PG2, PG4 and PG5, the reduction in the starch hydrolysis with increasing concentration of the protein is not as drastic as in cases of PG1 and PG3. In the case of PG2, a small reduction compared to PG1 implies that the first $30 \mathrm{~N}$ terminal residues of AbpA may bind to HSAmy in one or more of secondary saccharide binding sites of HSAmy. Moreover, starch binding is not affected in the presence of PG4 and PG5 since there is very little effect in the reduction in the residual activity. The starch hydrolysis data from PG1 and PG4 variants suggest that some of the residues in the C-terminal domain bind to HSAmy at sites where starch also binds. The absence of these residues did not affect starch binding and hence the hydrolytic efficiency of HSAmy is restored. Similarly, while the C-terminal residues might be binding to starch binding sites on the surface of HSAmy, the presence of only this domain (PG5) is ineffective to exhibit a reduction in starch hydrolytic activity. This is highly likely due to absence of any ordered secondary structures in PG5.

\section{Hydrolysis of oligosaccharides}

Unlike starch, which might bind to HSAmy at multiple secondary

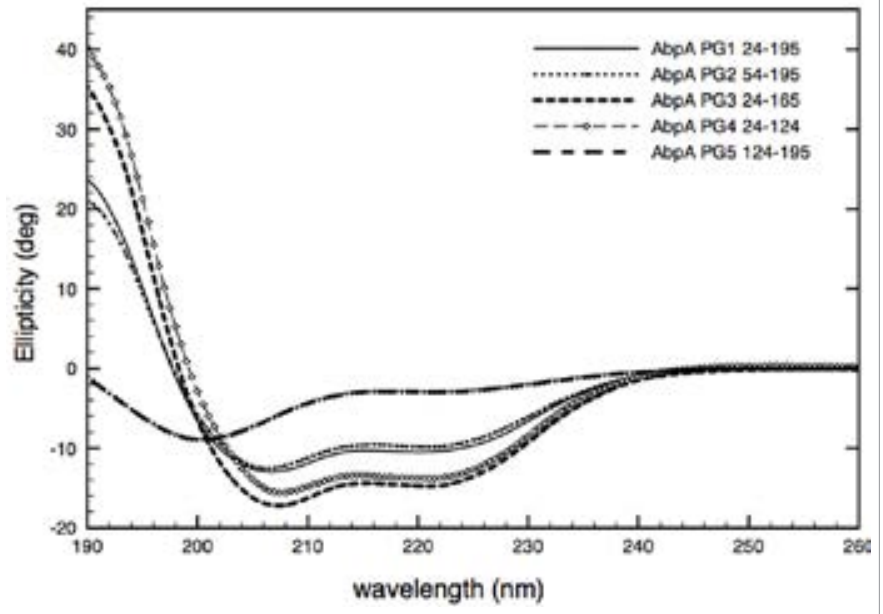

Figure 2: $C D$ spectra of AbpA proteins. The double minima in the spectra of the proteins PG1-PG4 are characteristic of proteins with significant secondary structures such as $\alpha$-helix and $\beta$-sheet. Note that PG3 and PG4 display an increase in the ellipticity at $222 \mathrm{~nm}$ suggesting that these structures have a higher $\alpha$-helix content. 


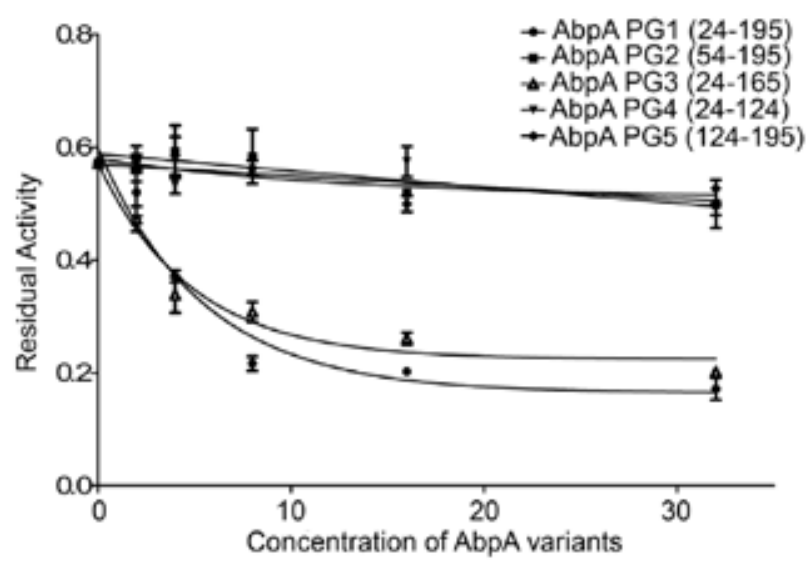

Figure 3: Starch hydrolysis by HSAmy bound to AbpA variants. The residual specific activities of the enzymes with and without $A b p A$ variants is a measure of the efficiency of AbpA binding as well as whether or not starch binding is impeded. A reduction in $y$-axis value for HSAmy indicates better binding to starch; $x$-axis show concentrations of AbpA in $\mu \mathrm{g} / \mathrm{ml}$ ranging from 2 to $32 \mu \mathrm{g}$ and $y$-axis show the residual starch hydrolysis activity.

saccharide binding sites, smaller oligosaccharides such as G5, G6 and G7 bind to the active site of HSAmy completely [16] Previous studies have shown that HSAmy possesses seven glucose-binding subsites [16], which completely fill the active site. In order to test whether or not the AbpA binds to one or more of the subsites of the active site, we tested the hydrolytic ability of HSAmy against maltoheptaose in the presence of AbpA and its variants. The standard hydrolysis curve of maltoheptaose was compared to the hydrolysis of maltoheptaose with the reaction mixture of AbpA and HSAmy (Figure 4). The hydrolysis pattern of maltoheptaose with HSAmy was identical to the pattern obtained for the mixtures of HSAmy and AbpA variants. These results suggest that AbpA binds to HSAmy by leaving the active site open for the hydrolysis of G7. Comparison of the hydrolytic abilities of HSAmy showed that G7 was hydrolyzed to the same extent in the presence of PG1, PG2 and PG3 while HSAmy hydrolyzed G7 to a larger extent in the presence of PG4 and PG5.

\section{Binding of AbpA variants to HSAmy using surface plasmon resonance spectroscopy}

A representative sensorgram depicting the binding of HSAmy $(0.5 \mu \mathrm{M})$ to the AbpA variants is shown in Figure 5. The binding efficiency was observed in the order PG1 $>$ PG3 $>$ PG2 $>>$ PG4. Clearly, the sensorgram corresponding to PG4 shows that there is very little binding. Because of the unordered nature of PG5 and insensitivity of HSAmy to the presence of PG5 in starch hydrolysis (Figure 3), PG5 was not used in the Biacore experiments. In addition, the binding of HSAmy to the immobilized AbpA variants resulted in a dosedependent increase in HSAmy binding for the mutants PG1, PG2 and PG3 (data not shown). Analysis of the binding data (BIAevaluation 3.2 software) indicated a weak affinity in the binding to PG2 coupled with a faster dissociation rate and a slower association rate (Table 1). In sharp contrast, the Kd for HSAmy binding to PG1 and PG3 were comparable (same order of magnitude) and suggests a very tight binding between HSAmy: PG1 and HSAmy: PG3. Interestingly, the absence of the Cterminal residues in PG3 does not affect the binding to HSAmy whereas the absence of the $\mathrm{N}$ - terminal residues in PG2 (residues 24-53) does affect the binding. However, PG4 which lacks the C-terminal domain (last 60 residues) completely lost its ability to bind HSAmy suggesting that residues 124-165 are essential to bind to HSAmy. Taken together, the results of these experiments suggest that some or all of residues 24-
53 at the $\mathrm{N}$-terminus and residues $124-165$ at the $\mathrm{C}$-terminal end might play a role in HSAmy interaction.

\section{Discussion}

\section{CD spectra of AbpA variants}

The CD spectra in the far-UV region are well known to reflect the secondary structure of proteins, although the tertiary structures have been known to influence the spectrum sometimes due to the interaction among aromatic residues. The mature sequence of $\mathrm{AbpA}$ does not contain many aromatic residues except a few in the $\mathrm{N}$-terminal end and a very few at the C-terminal end. Thus, the observation of the typical spectrum corresponding to an $\alpha$ - helical protein is representative of the structure adopted by the molecule. Successive deletion of residues from the C-terminal end appears to increase the ratio of the helix vs. strand and other structures as the ellipticity value at $222 \mathrm{~nm}$, a measure sometimes used to represent helicity increases in PG3. The structural

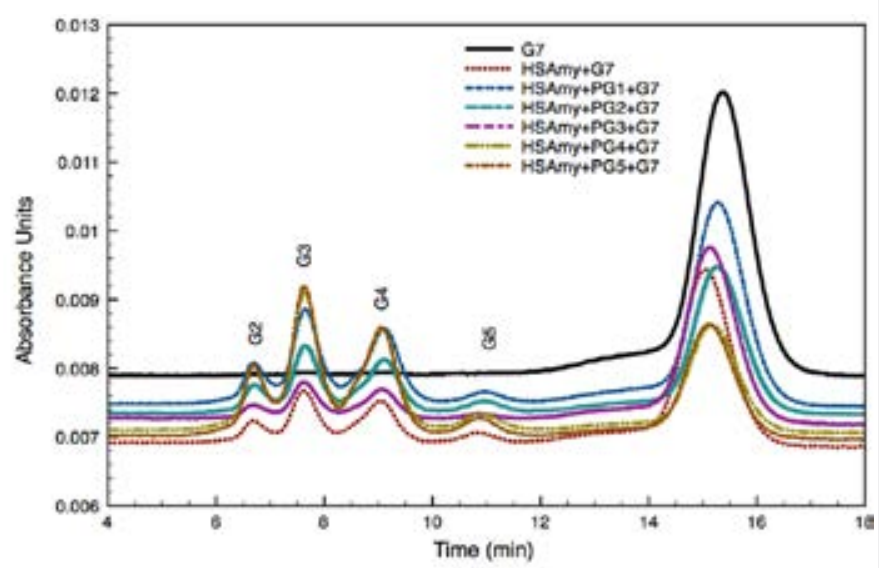

Figure 4: Hydrolytic action profiles of the HSAmy in the presence of AbpA variants. The action patterns are identical for the mutants, albeit differences in the amount of products generated.

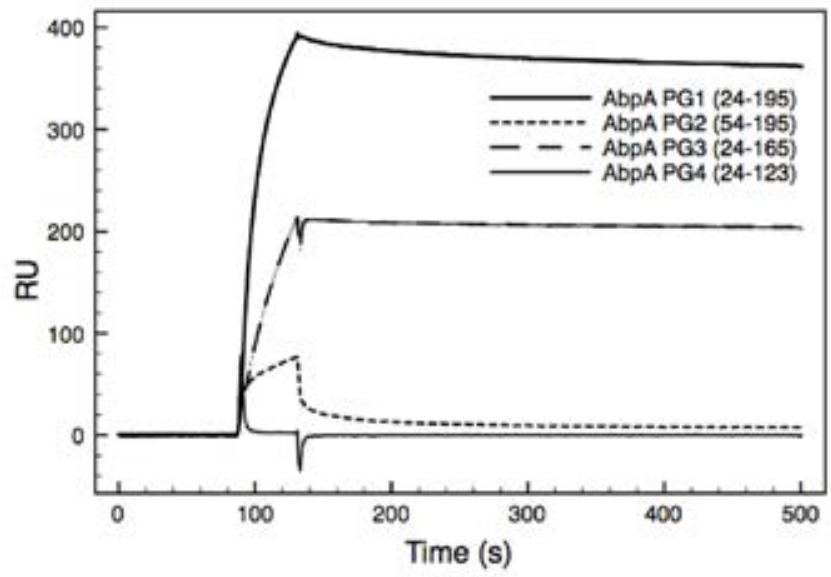

Figure 5: Binding of HSAmy to AbpA variants. Sensorgrams comparing the binding of HSAmy to AbpA wherein HSAmy was supplied at $0.5 \mu \mathrm{M}$ concentration in the mobile phase. The binding curves were similar for concentrations of HSAmy at 0.125 and $0.25 \mu \mathrm{M}$ (not shown). The curves were adjusted for the effects of buffers and salts on resonance signals using the uncoated (blank) surface in flow cell 1 as reference. HSAmy shows a similar association and slow dissociation curves with PG1 and PG3 whereas with PG2 it shows quick association and dissociation curves. Note that there is no association curve with PG4. 
model obtained for AbpA (Figure 1) is somewhat limited in the absence of a definitive three-dimensional structure of AbpA. Nevertheless, it could be argued that the hypothetical model derived for AbpA has some validity since the results obtained through $\mathrm{CD}$ analyses support the preponderance of the helical nature obtained for the model.

\section{Significance of the designed selective deletions and interaction between AbpA and HSAmy}

Earlier structural studies have shown that HSAmy possesses multiple secondary oligosaccharide binding sites, which influence the binding to AbpA [20]. It was also noted that there exists similarity between bacterial binding sites and substrate-binding sites [26]. An interesting outcome of the combination of these studies is that both starch binding and AbpA binding to HSAmy might use multiple saccharide binding sites located on the surface of HSAmy. A further outcome of the recent study [20] on the role of aromatic residues in bacterial binding was that two aromatic residues (W203 and W284) might play a role in bacterial binding activity. Our results suggesting that both the $\mathrm{N}$-terminal and the $\mathrm{C}$-terminal helices in the hypothetical model of AbpA might be involved in binding to HSAmy is particularly interesting in this regard. If the model of $\mathrm{AbpA}$ is relevant for this discussion, it should be noted that the helix consisting of residues 34-45 (NDGAYYLQFT) has two Tyr residues. When this segment is absent (PG2), then the SPR data suggest that there is weak affinity to HSAmy. It is likely that aromatic residues might dominate the interaction between HSAmy and AbpA. In this regard, PG4 also has lost its ability to bind HSAmy when the C-terminal residues (138-150) are absent (Figure 5). This segment contains two Tyr residues (Tyr129 and Tyr142), one of which occurs in a $\beta$-strand (TYIQ) and the second one in a helical segment (ANARYLKRVGAAN) further suggesting that aromatic residues in AbpA might play a role in the binding to HSAmy. It is tempting to speculate that these spatially well-separated aromatic residues in AbpA might be suitably juxtaposed to bind to the secondary saccharide sites harboring W203 and W284 in HSAmy. However, proof for this requires the validation of the model by $\mathrm{x}$-ray structure analysis or other types of three-dimensional structure determination.

\section{Conclusion}

We show here that the AbpA might adopt a mostly helical structure and two segments in AbpA when absent decrease the binding to HSAmy. The presence of aromatic residues in these segments points to the potential role of these residues in the binding interaction paralleling the role of aromatic residues on the surface of HSAmy in binding to AbpA.

\section{Acknowledgements}

The authors would like to thank the New Jersey Health Foundation and the Department of Oral Biology, NJDS, UMDNJ for financial support. The assistance provided by Dr. Sangeetha Purushotham and Ms. Manisha Patel at University of Alabama at Birmingham is greatly appreciated in the use of the BIAcore facility.

\section{References}

1. Nikitkova AE, Haase EM, Scannapieco FA (2013) Taking the starch out of oral biofilm formation: molecular basis and functional significance of salivary a-amylase binding to oral streptococci. Appl Environ Microbiol 79: 416-423.

2. Yao Y, Grogan J, Zehnder M, Lendenmann U, Nam B, et al. (2001) Compositional analysis of human acquired enamel pellicle by mass spectrometry. Arch Oral Biol 46: 293-303

3. Douglas CW (1983) The binding of human salivary alpha-amylase by ora strains of streptococcal bacteria. Arch Oral Biol 28: 567-573.

4. Scannapieco FA, Bergey EJ, Reddy MS, Levine MJ (1989) Characterization of salivary alpha-amylase binding to Streptococcus sanguis. Infect Immun 57: 2853-2863.

5. Douglas CW, Pease AA, Whiley RA (1990) Amylase-binding as a discriminator among oral streptococci. FEMS Microbiol Lett 54: 193-197.

6. Kilian M, Nyvad B (1990) Ability to bind salivary alpha-amylase discriminates certain viridans group streptococcal species. J Clin Microbiol 28: 2576-2577.

7. Brown AE, Rogers JD, Haase EM, Zelasko PM, Scannapieco FA (1999) Prevalence of the amylase-binding protein $A$ gene (abpA) in oral streptococci. J Clin Microbiol 37: 4081-4085.

8. Scannapieco FA, Haraszthy GG, Cho MI, Levine MJ (1992) Characterization of an amylase-binding component of Streptococcus gordonii G9B. Infect Immun 60: 4726-4733.

9. Gwynn JP, Douglas CW (1994) Comparison of amylase-binding proteins in ora streptococci. FEMS Microbiol Lett 124: 373-379.

10. Rogers JD, Haase EM, Brown AE, Douglas CW, Gwynn JP, et al. (1998) Identification and analysis of a gene (abpA) encoding a major amylase-binding protein in Streptococcus gordonii. Microbiology 144 : 1223-1233.

11. Rogers JD, Palmer RJ Jr, Kolenbrander PE, Scannapieco FA (2001) Role of Streptococcus gordonii amylase-binding protein A in adhesion to hydroxyapatite, starch metabolism, and biofilm formation. Infect Immun 69: 7046-7056.

12. Chaudhuri B, Rojek J, Vickerman MM, Tanzer JM, Scannapieco FA (2007) Interaction of salivary alpha-amylase and amylase-binding-protein A (AbpA) of Streptococcus gordonii with glucosyltransferase of $\mathrm{S}$. gordonii and Streptococcus mutans. BMC Microbiol 7: 60 .

13. Li L, Tanzer JM, Scannapieco FA (2002) Identification and analysis of the amylase-binding protein $\mathrm{B}(\mathrm{AbpB})$ and gene $(\mathrm{abpB})$ from Streptococcus gordonii. FEMS Microbiol Lett 212: 151-157.

14. Ramasubbu N, Paloth V, Luo Y, Brayer GD, Levine MJ (1996) Structure of human salivary alpha-amylase at $1.6 \mathrm{~A}$ resolution: implications for its role in the oral cavity. Acta Crystallogr D Biol Crystallogr 52: 435-446.

15. Mishra PJ, Ragunath C, Ramasubbu N (2002) The mechanism of salivary amylase hydrolysis: role of residues at subsite S2'. Biochem Biophys Res Commun 292: 468-473.

16. Ramasubbu N, Ragunath C, Mishra PJ (2003) Probing the role of a mobile loop in substrate binding and enzyme activity of human salivary amylase. $\mathrm{J} \mathrm{Mol} \mathrm{Biol}$ 325: 1061-1076.

17. Remenyik J, Ragunath C, Ramasubbu N, Gyémánt G, Lipták A, et al. (2003) Introducing transglycosylation activity into human salivary alpha-amylase (HSA). Org Lett 5: 4895-4898.

18. Ramasubbu N, Ragunath C, Mishra PJ, Thomas LM, Gyémánt G, et al. (2004) Human salivary alpha-amylase $\operatorname{Trp} 58$ situated at subsite -2 is critical for enzyme activity. Eur J Biochem 271: 2517-2529.

19. Ramasubbu N, Sundar K, Ragunath C, Rafi MM (2004) Structural studies of Phe256Trp mutant of human salivary alpha-amylase: implications for the role of a conserved water molecule in enzyme activity. Arch Biochem Biophys 421 $115-124$.

20. Ragunath C, Manuel SG, Venkataraman V, Sait HB, Kasinathan C, et al. (2008) Probing the role of aromatic residues at the secondary saccharide-binding sites of human salivary alpha-amylase in substrate hydrolysis and bacterial binding J Mol Biol 384: 1232-1248.

21. Dubendorff JW, Studier FW (1991) Creation of a T7 autogene. Cloning and expression of the gene for bacteriophage T7 RNA polymerase under control of its cognate promoter. J Mol Biol 219: 61-68.

22. Söding J, Biegert A, Lupas AN (2005) The HHpred interactive server for protein homology detection and structure prediction. Nucleic Acids Res 33: W244-248.

23. Jones DT (1999) Protein secondary structure prediction based on positionspecific scoring matrices. J Mol Biol 292: 195-202.

24. Sali A, Blundell TL (1993) Comparative protein modelling by satisfaction of spatial restraints. J Mol Biol 234: 779-815.

25. DeLano WL (2002) The PyMOI User's Manual, Delano Scientific, San Carlos, CA

26. Scannapieco FA, Bhandary K, Ramasubbu N, Levine MJ (1990) Structura relationship between the enzymatic and streptococcal binding sites of human salivary alpha-amylase. Biochem Biophys Res Commun 173: 1109-1115. 